\title{
Scroll-wave dynamics in the presence of ionic and conduction inhomogeneities in an anatomically realistic mathematical model for the pig heart
}

\author{
R. Majumder ${ }^{a}$, Rahul Pandit ${ }^{b}$, A. V. Panfilov ${ }^{c, *}$ \\ ${ }^{a}$ Laboratory of Experimental Cardiology, Dept. of Cardiololgy, Heart Lung Center, Leiden University Medical center, 2333ZA Leiden, \\ the Netherlands \\ ${ }^{b}$ Centre for Condensed Matter Theory, Dept. of Physics, Indian Institute of Science, Bangalore 560012, India; Jawaharlal Nehru \\ Centre for Advanced Scientific Research, Bangalore, India.
}

\author{
${ }^{c}$ Dept. of Physics and Astronomy, Gent University, Krijgslaan 281, S9, 9000 Gent, Belgium; Moscow Institute of Physics and \\ Technology, (State University), Dolgoprudny, Moscow Region, Russia \\ *e-mail: Alexander.Panfilov@ugent.be
}

Submitted 22 August 2016

\begin{abstract}
Nonlinear waves of the reaction-diffusion (RD) type occur in many biophysical systems, including the heart, where they initiate cardiac contraction. Such waves can form vortices called scroll waves, which result in the onset of life-threatening cardiac arrhythmias. The dynamics of scroll waves is affected by the presence of inhomogeneities, which, in a very general way, can be of (i) ionic type, i.e., they affect the reaction part, or (ii) conduction type, i.e., they affect the diffusion part of an RD equation. We demostrate, for the first time, by using a state-of-the-art, anatomically realistic model of the pig heart, how differences in the geometrical and biophysical nature of such inhomogeneities can influence scroll-wave dynamics in different ways. Our study reveals that conduction-type inhomogeneities become increasingly important at small length scales, i.e., in the case of multiple, randomly distributed, obstacles in space at the cellular scale $(0.2-0.4 \mathrm{~mm})$. Such configurations can lead to scroll-wave break up. In contrast, ionic inhomogeneities, affect scroll-wave dynamics significantly at large length scales, when these inhomogeneities are localized in space at the tissue level $(5-10 \mathrm{~mm})$. In such configurations, these inhomogeneities can (a) attract scroll waves, by pinning them to the heterogeneity, or (b) lead to scroll-wave breakup.
\end{abstract}

Introduction: Nonlinear waves occur in excitable media of physical, chemical, and biological origin. Such waves can form vortices in two and three dimensions; these are called spiral and scroll waves, respectively, and they are involved in the spatiotemporal organization of wave dynamics in various complex systems. Therefore, the study of such waves is a subject of interest in a broad area of research. One of the most important applications of such studies is the formation of vortices in cardiac tissue, which is associated with the onset and devel'opment of lethal cardiac arrhythmias 1, 2, 3, 4, 5, 6, 7. Thus, understanding the factors that determine the dynamics of scroll waves is a topic of great interest. Cardiac arrhythmias, such as ventricular tachycardias (VT) are generally associated with stationary, meandering, or drifting, periodic or quasiperiodic scroll waves; whereas, ventricular fibrillation (VF) is associated with scrollwave break up. The dynamical behaviour of scroll waves in cardiac tissue is affected significantly by the presence of inhomogeneities $[8,9,10,11,2,3,12,13,14,15,16$, which can occur in the heart in many forms. However, biophysically, they can be grouped into two major classes: (i) Ionic-type, i.e., inhomogeneities in the properties of different cells that constitute the system; and (ii) conduction-type, i.e., inexcitable obstacles. An indepth knowledge of the role of these inhomogeneities is essential for understanding the mechanisms that underlie most cardiac arrhythmias.

In experiments, it is often difficult to study systematically the role of these inhomogeneities in the development of arrhythmias, with regard to the nature, position, and distribution of these inhomogeneities within the heart. Thus, it is important to search for alternative methods of investigation. Mathematical modelling provides an important tool here; it has been used extensively, with outstanding success, in interdisciplinary science. From a mathematical point of view, the excitable, cardiac-tissue medium is described by a reaction-diffusion (RD) equation of the type:

$$
\frac{\partial v}{\partial t}=\nabla \cdot(\mathcal{D} \nabla v)+\mathcal{F}(g, v),
$$

with the reaction part $\mathcal{F}(g, v)$ accounting for properties of cardiac cells and the diffusion part $\nabla \cdot \mathcal{D} \nabla v$, for the connection of cells to tissue. In this setting, an ionic 
inhomogeneity represents a modification of $\mathcal{F}$, whereas a conduciton inhomogeneity involves a modification of $\nabla \cdot \mathcal{D} \nabla v$.

In this Letter, we present an extensive numerical study of scroll-wave dynamics in the presence of inhomogeneities in an anatomically realistic model of the pig heart. We have used the single-cell, modified, Luo-Rudy I (mLRI) model 17 to construct our cardiac-tissue model and the anatomically realistic geometry obtained in [18. We have studied the effects on scroll-wave dynamics of (i) large-length-scale, solitary inhomogeneity (old infarction) and (ii) small-length-scale, multiple, conduction inhomogeneities (fibrosis) and compared our results from these studies with those we have obtained from similar (i) large- and (ii) small-length-scale ionic inhomogeneities. Our results illustrate, for the first time, that conduction inhomogeneities influence scroll-wave dynamics significantly, when they occur at small length scales (sub-millimeter) in distributed patterns; by contrast, ionic inhomogeneities play a significant role in influencing such dynamics at large length scales (millimeters), when they are localized in space.

Methods: A modified version of the original LuoRudy I model [19], namely, the mLRI [17], was used to model the electrophysiological properties of the pig cardiac cell. The original parameters of the mLRI model, including the effects of Eqs.4-7 of Qu, et al. [17] were used to simulate the pig heart electrophysiology in our studies. In two dimensions (2D), this parameter set yielded a spiral wave rotating at a frequency $\simeq 12 \mathrm{~Hz}$, the approximate frequency of spiral waves [20, 21] in the pig heart.

Here the transmembrane potential $(V)$ of a cardiac cell depends on the sum of 6 ionic currents $\left(I_{\text {ion }}\right)$ and the applied current stimulus $\left(I_{\text {stim }}\right)$ according to the following partial differential equation:

$$
\frac{\partial V}{\partial t}=\nabla \cdot(\mathcal{D} \nabla V)-\frac{I_{\text {ion }}+I_{\text {stim }}}{C},
$$

where $C$ is the specific membrane capacitance of the cell. The diffusion tensor $\mathcal{D}$ is a $3 \times 3$ matrix [22, 23] with elements

$$
\mathcal{D}_{i j}=D_{\|} * \delta_{i j}+\left(D_{\|}-D_{\perp}\right) \alpha_{i} \alpha_{j} .
$$

Diffusion coefficients for longitudinal $\left(D_{\|}\right)$and transverse $\left(D_{\perp}\right)$ propagation are chosen as $0.001 \mathrm{~cm}^{2} / \mathrm{ms}$ $0.00025 \mathrm{~cm}^{2} / \mathrm{ms}$, respectively, to obtain conduction velocities $\simeq 59 \mathrm{~cm} / \mathrm{s}$ and $\simeq 20.5 \mathrm{~cm} / \mathrm{s}$, respectively, in the longitudinal and transverse directions; these are consistent with the normally accepted values for pig cardiac tissue 24. The vector $\alpha$ specifies the local, muscle-fiber orientation.

To construct an anatomically realistic simulation domain, processed DTMRI data points, have been embedded into a cubical simulation domain 22, 23, with $328^{3}$ vertices. Each node in this cubical domain are labeled as a heart point (HP), if the node coincides with one of the points from the processed data set, or as a non-heart point (NHP) otherwise. The temporal part of Eq. 2 is solved by using Euler's method; we use a centered, finite-difference scheme with $\delta x=\delta y=\delta z=0.025 \mathrm{~cm}$ to solve Eq. 2 in space. Zero-flux boundary conditions are incorporated on the boundaries of the anatomically realistic heart by adopting a phase-field approach [25].

To model large-scale inhomogeneities, spheres of radius $10 \delta x$ were embedded in 3 different positions of the simulated heart wall $(P 1, P 2$, and $P 3)$, with the possibility of protrusion into the inner cavities, or out of the exterior surface of the heart. Small-scale inhomogeneities were modeled as randomly distributed cubical cells of side $1 \delta x$ [23, that contained 1 node each ( $5 \%, 10 \%, 15 \%$, and $20 \%$ by number). To model conduction-type inhomogeneities, $D_{\|}=D_{\perp}$ was set to 0 inside the inhomogeneity. To model ionic inhomogeneities, only the value of the slow, inward conductance $G_{s i}$ was set to $0.02 \mathrm{mS} / \mathrm{cm}^{2}$ [3] at the sites covered by the inhomogeneity, without adjusting the elements of the diffusion tensor. Figure 1 shows the positions and distributions of inhomogeneities considered.
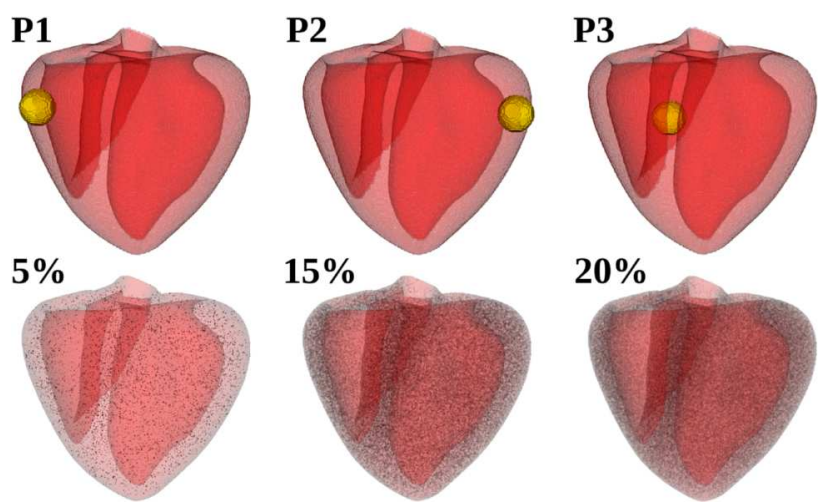

Figure 1. The upper panel shows schematic diagrams of the pig heart with the large-length-scale, spherical inhomogeneity at positions P1, P2, and P3. The lower panel shows small-length-scale inhomogeneities that are distributed randomly, covering 5\%, $15 \%$, and $20 \%$, respectively, of nodal sites.

Results: No inhomogeneities: In the absence of inhomogeneities, we obtain a single stable periodically rotating scroll, with an average frequency $12 \mathrm{~Hz}$. We 
then study scroll-wave dynamics in the presence of large- and small-scale conduction and ionic inhomogeneities. Thus, in total, we consider 10 different cases with different inhomogeneities. Our main findings from these 10 cases are listed in Tables I \& II. The details of our results are also discussed below with figures to illustrate the most important types of dynamical behaviours.

Conduction Inhomogeneities: Figure 2 shows the effects of various conduction heterogeneities on scroll-wave dynamics (cases 1-2). We find that solitary, large-scale conduction inhomogeneities do not have any pronounced effect on scroll-wave dynamics. Indeed, at all 3 positions of the inhomogeneity $P 1, P 2$ and $P 3$, the scroll wave remains insensitive to the presence of the obstacle (Figures 2 (a)). However, small-scale conduction inhomogeneities affect scroll-wave dynamics substantially by changing the characteristics of the scroll wave and causing its breakup. At all distributions of small-scale conduction inhomogeneities that we have considered, namely, 5\%,10\%,15\% and $20 \%$ inhomogeneity (Case 2), we observe the following: (i) a shortening of the spatial wavelength of the scroll, and (ii) scroll-wave breakup at inhomogeneities $\gtrsim 15 \%$ (Figure 2 (b)).

\begin{tabular}{|l|l|l|}
\hline \multicolumn{3}{|c|}{ Table I: Conduction inhomogeneity } \\
\hline $\begin{array}{l}\text { Case } \\
\text { no. }\end{array}$ & $\begin{array}{l}\text { Inhomogeneity } \\
\text { type }\end{array}$ & Dynamics \\
\hline 1 & $\begin{array}{l}\text { large }(P 1) \\
\text { large }(P 2)\end{array}$ & $\begin{array}{l}\text { Scroll wave remains passive } \\
\text { towards the presence of the } \\
\end{array}$ \\
large $(P 3)$ & inhomogeneity. \\
\hline 2 & small $(5 \%)$ & Scroll wavelength reduces. \\
& small $(10 \%)$ & Scroll wavelength reduces. \\
& small $(15 \%)$ & Unstable breakup. \\
& small $(20 \%)$ & Stable breakup. \\
\hline
\end{tabular}

Ionic Inhomogeneities: Figure 3 illustrates the effects of various ionic heterogeneities on scrollwave dynamics (Cases 3-4). We see that solitary, large-scale ionic inhomogeneities have a substantial effect on scroll-wave dynamics. We observe interesting dynamical behaviour, such as, scroll-wave breakup (Figure [3(a):P1) and anchoring P3) (Case 3). On the contrary, small-scale ionic inhomogeneities do not lead to qualitatively interesting dynamics: for all the inhomogeneities we have considered, namely, $5 \%, 10 \%, 15 \%$ and 20\% (Case 4) (Figure 3 (b)) we do not find a pronounced change in scroll-wave dynamics.

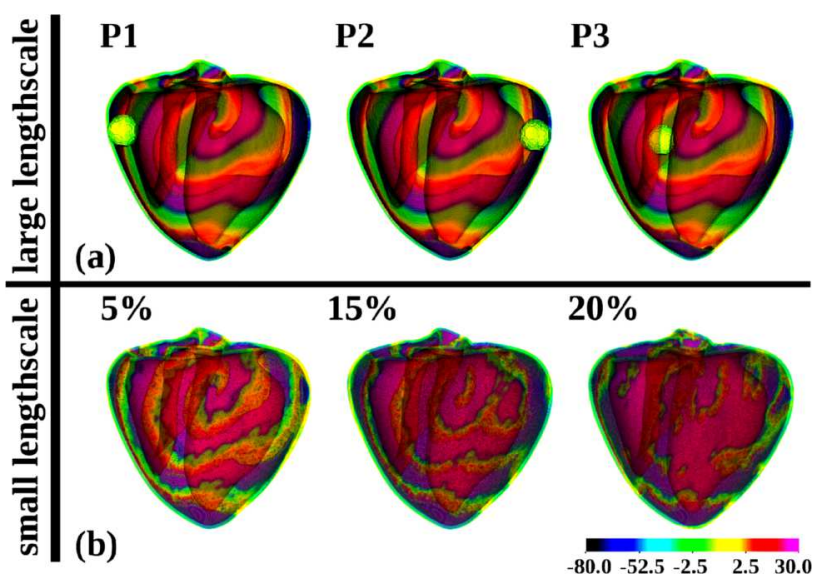

Figure 2 Representative snapshots of scroll-wave dynamics in anatomically realistic pig hearts in the presence of (a) large- and (b) small-scale (b), conduction inhomogeneities.

\begin{tabular}{|l|l|l|}
\hline \multicolumn{3}{|c|}{ Table II: Ionic inhomogeneity } \\
\hline $\begin{array}{l}\text { Case } \\
\text { no. }\end{array}$ & $\begin{array}{l}\text { Inhomogeneity } \\
\text { type }\end{array}$ & Dynamics \\
\hline 3 & $\begin{array}{l}\text { large }(P 1) \\
\text { large }(P 2) \\
\text { large }(P 3)\end{array}$ & $\begin{array}{l}\text { Stable breakup. } \\
\text { No change. } \\
\text { Stable anchoring. }\end{array}$ \\
\hline 4 & $\begin{array}{l}\text { small }(5 \%) \\
\text { small }(10 \%) \\
\text { small }(15 \%) \\
\text { small }(20 \%)\end{array}$ & $\begin{array}{l}\text { No significant qualitative } \\
\text { change. Dynamics is } \\
\text { insensitive to the presence } \\
\text { of the inhomogeneity. }\end{array}$ \\
\hline
\end{tabular}

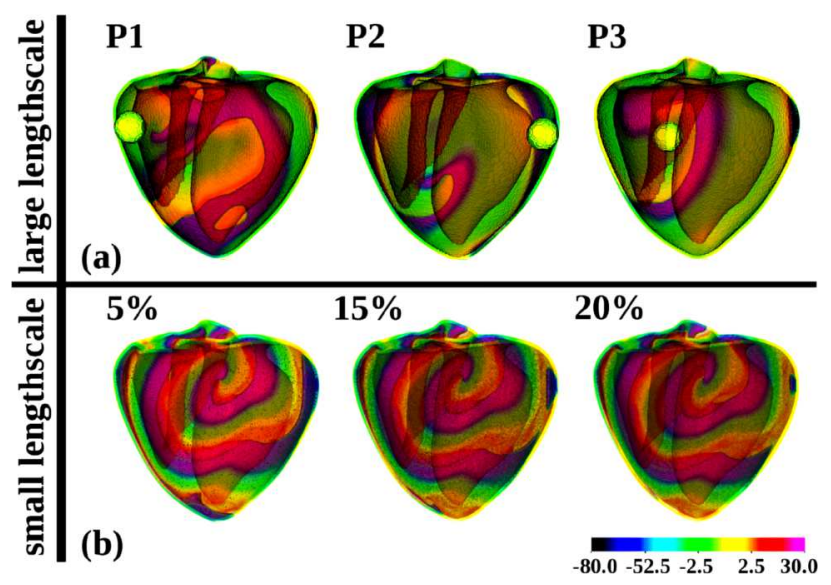

Figure 3 Representative snapshots of scroll-wave dynamics in anatomically realistic pig hearts in the presence of (a) large- and (b) small-scale (b) ionic inhomogeneities.

Taken together, our results demonstrate that large-scale conduction inhomogeneities do not affect scroll-wave dynamics in the pig heart. However, if the 
inhomogeneity is of ionic type, it can lead to scroll-wave breakup. On the contrary, small-scale conduction inhomogeneities have a significant influence on the dynamics of scroll waves; such inhomogeneities generally lead to some decrease in the spatial wavelength of the scroll wave and initiate scroll-wave breakup. Small-scale ionic inhomogeneities, however, prove to be protective against breakup.

Discussion:We have carried out a comprehensive numerical study of scroll-wave dynamics in an ionic model for pig cardiac tissue; and we have compared, in the same conditions, the effects of conduction and ionic heterogeneities, both for small and large length scales [3, 12, 13, on such scroll-wave dynamics.

Our principal, qualitative result that small-scale inhomogeneities are important in the diffusion part is a consequence of the effect of the diffusion processes on the reaction part (called the electrotonic effect in electrophysiology) [26. However, we have also found that small-scale conduction inhomogeneities are not averaged out by the diffusion. Therefore, their mean-field consideration, e.g., by using homogenization techniques, should be done with caution. For large-scale heterogeneities, our results are in line with findings for human cardiac-tissue simulations [27; however, these have been performed on a completely different cardiac geometry, different cell models, and for substantially different values of scroll wavelengths. In addition to dynamical anchoring (via the transient-breakup phase) described in Ref. 27] we have also observed anchoring of the other type resulting from a drift of the scroll for qualitatively different positions of the heterogeneity: in particular, we have placed heterogeneity inside the septum and have found that it can attract scroll waves and thus lead to interesting new dynamics.

The work of RM and RP was supported by DST, UGC, and CSIR (India). RM and RP would like to thank SERC (IISc) for computational resources. The work of AVP was supported by the Research Foundation-Flanders (FWO Vlaanderen).

1. Clayton RH, Bernus O, Cherry EM, Dierckx H, Fenton FH, et al. Prog Biophys Mol Biol. 104, 13:2248 (2011).

2. Shajahan TK, Sinha S, and Pandit R, Phys. Rev. E. 75:011929-1 - 011929-8 (2007).

3. Shajahan TK, Nayak AR, and Pandit R, PLoS ONE. 4(3):e4738 (2009).

4. Jalife J, Gray RA, Morley GE, and Davidenko JM, Chaos. 8, 1:79-93 (1998).

5. Mann DL, Circ. Res. 91:988-998 (2002).
6. Kléber AG, Rudy Y, Physiol. Rev. 84:431-488 (2004).

7. Vigmond EJ, Weber dos Santos R, Prassl AJ, Deo M, Plank G, Prog Biophys Mol Biol. 96:3-18 (2008).

8. Ikeda T, Yashima M, Uchida T, Hough D, Fishbein MC, et al. Circ. Res. 81:753 (1997).

9. Valderr/'abano M, Kim YH, Yashima M, Wu TJ, Karagueuzian HS, et al. J Am Coll Cardiol. 36: 2000 (2000).

10. Lim ZY, Maskara B, Aguel F, Emokpae Jr. R, Tung L, Circulation. 114:2113-2121 (2006).

11. Davidenko JM, Pertsov AV, Salomonsz R, Baxter W, and Jalife J, Nature. 355:349 (1992).

12. Majumder R, Nayak AR, Pandit R, PLoS ONE. 6(4):e18052 (2011).

13. Majumder R, Nayak AR, Pandit R, PLoS ONE. 7(10):e45040 (2012).

14. Nayak AR, Shajahan TK, Panfilov AV, and Pandit R, PLoS ONE. 8(9):e72950 (2013).

15. Rudenko AN, Panfilov AV, Studia Biophysica. 98:183188 (1983).

16. Panfilov AV, Vasiev BN, Physica D.49:107-113, (1991).

17. Qu Z, Weiss JN, and Garfinkel A, Am J Physiol Heart Circ Physiol. 276:269-283 (1999).

18. Stevens C, Remme E, LeGrice IJ, Hunter PJ, J Biomech. 36:737-748 (2003).

19. Luo CH, Rudy Y, Circ. Res. 68:1501-1526 (1991).

20. Panfilov AV, Heart Rhythm. 3(7):862-864 (2006).

21. Newton JC, Smith WM, Ideker RE, Circ. Res. 94:836842 (2004).

22. Clayton RH, and Panfilov AV, Prog Biophys Mol Biol. 96:19-43 (2008).

23. Ten Tusscher KHWJ, Panfilov AV, Europace. 9:v138v145, (2007).

24. Kléber A, Janse MJ, Wilms-Schopmann FJG, Wilde AAM, and Coronel R, Circulation 73(1):189-198 (1986).

25. Fenton FH, Cherry EM, Karma A, and Rappel WJ, Chaos 15, 013502 (2005).

26. Defauw A, Kazbanov IV, Dierckx H, Dawyndt P, Panfilov AV, PLoS One. 8(11):e79607/1-e79607/12, (2013).

27. Defauw A, Vandersickel N, Dawyndt P, Panfilov AV, Am J Physiol Heart Circ Physiol. 307:H1456-68, (2014). 